\title{
Ecologías sensibles. Arte de enfoque colaborativo y crisis ecosocial en el contexto americano
}

\section{Sensible ecologies. Collaborative art and ecosocial crisis in the American context}

TIPO DE TRABAJO: Comunicación.

PALABRAS CLAVE

Arte, ecología, colaboración, crisis ecosocial, invisibilización.

KEY WORDS

Art, ecology, collaboration, ecosocial crisis, invisibilization.

RESUMEN

La presente comunicación recoge los resultados parciales de una estancia de investigación predoctoral realizada en el Center for Creative Ecologies de la University of California Santa Cruz, Estados Unidos. Partiendo de un análisis de los múltiples niveles de invisibilización de la crisis ecosocial contemporánea (Herrero 2013; Riechmann 2011), se exploran las intersecciones entre formas de arte vinculadas a la ecología y procesos colaborativos de creación.

En concreto, haciendo referencia al potencial del arte para reconfigurar lo sensible y las formas de visibilidad (Rancière 2007) junto a su capacidad de abordar temas complejos como las problemáticas ambientales desde una perspectiva postantropocéntrica (Demos 2017), se examina el trabajo de artistas y colectivos actualmente activos en el continente americano como Carolina Caycedo (Colombia), The Harrison Studio (Estados Unidos) y Desert Art Lab (Estados Unidos). A través del análisis crítico de proyectos artísticos y entrevistas semiestructuradas con los artistas, se estudian la intencionalidad de los creadores y las diferentes estrategias colaborativas empleadas para hacer visibles los impactos del modelo extractivista, las luchas sociales para la preservación de los ecosistemas naturales y de los saberes indígenas y los procesos de resiliencia y adaptación de los organismos no-humanos a las alteraciones antropogénicas del clima.

El contexto geopolítico escogido resulta particularmente significativo por la pervasividad del paradigma neoliberal y por la consecuente erosión de derechos, tanto sociales como ambientales, que lo ha caracterizado en las últimas décadas. Al mismo tiempo la presencia de movimientos contraculturales vinculados a la ecología, al feminismo y a la defensa de las culturas nativas de las Américas ha permitido el emerger de otras sensibilidades y modelos de convivencia que han alimentado e inspirado las expresiones artísticas estudiadas.

\section{ABSTRACT}

This paper includes the partial findings of a research stay held at the Center for Creative Ecologies of the University of California Santa Cruz (U.S.A.). Starting from an analysis of the multiple forms of invisibilization of the contemporary ecosocial crisis (Herrero 2013, Riechmann 2011), I explore the intersections between ecology-related art forms and collaborative processes.

In particular, referring to the potential of art to reconfigure the sensible and distribute forms of visibility (Rancière 2008) together with its ability to address complex issues such as environmental and from a post-anthropocentric perspective (Demos 2017), I examine the work of artists and groups currently active in the Americas such as Carolina Caycedo (Colombia), The Harrison Studio (United States) and Desert Art Lab (United States). Through a critical analysis of their artistic projects and semi-structured interviews with artists and curators, I study the artists' intentionality and the different collaborative strategies they use to make the impacts of the extractivist 
model visible together with the social struggles for the preservation of ecosystems and indigenous knowledge systems, and the processes of resilience and adaptation of non-human organisms to anthropogenic climate change.

The chosen geopolitical context is especially significant because of the pervasiveness of the neoliberal paradigm that has characterized it in recent decades, with the consequent erosion of both social and environmental rights. At the same time, the presence of countercultural movements linked to ecology, feminism and the defense of the native cultures of the Americas has allowed the emergence of alternative discourses and models of coexistence that have nurtured and inspired the artistic manifestations our study revolves around.

\section{INTRODUCCIÓN}

La reciente proliferación de prácticas artísticas vinculadas a temas socioecológicos en el panorama contemporáneo refleja la creciente preocupación por las cuestiones ambientales en la sociedad actual. Los últimos informes sobre el estado del planeta y los efectos del cambio climático antropogénico describen escenarios dramáticos y preven, en ausencia de medidas urgentes y eficaces a nivel global, la desestabilización de los ecosistemas naturales y de los sistemas socioeconómicos y culturales que de ellos dependen (MassonDelmotte et al., 2018). Asistimos por lo tanto a una crisis que podría definirse como ecosocial por el inextricable vínculo entre los desequilibrios ecosistémicos y el deterioro de las condiciones de convivencia social. Sin embargo, a pesar de la magnitud de la problématica, las dimensiones reales de esta crisis raras veces son abordadas en su complejidad y calado en los discursos políticos y mediáticos dominantes. Al respecto, la antropóloga Yayo Herrero analiza, desde una perspectiva ecofeminista, los límites del modelo económico y cultural neoliberal señalando la sistemática invisibilización no solo de la relación de interdependencia entre sociedades humanas y ecosistemas naturales sino también de "la importancia central de los vínculos y de las relaciones para los seres humanos" y para la supervivencia de la especie (Herrero, 2013, p. 281). Asimismo, el filósofo Jorge Riechmann sostiene que las tácticas del sistema neoliberal "se orientan a invisibilizar, fragmentar y desconectar" (Riechmann, 2011, p. 41) con el objetivo de ocultar las externalidades sociales y ambientales de la economía capitalista, esquivar responsabilidades y neutralizar posibles formas de resistencia.

La presente investigación explora el papel de la creación artística en el cuestionamiento del paradigma neoliberal. En particular, nos interesa analizar de qué manera las practicas artísticas pueden contribuir a introducir en el debate público y en la sensibilidad colectiva cuestiones como la justicia climática o la construcción de futuros más ecológicos y equitativos. Nos centraremos en las intersecciones entre arte contemporáneo relacionado con la ecología y procesos colaborativos de creación. Aunque el giro ecológico (Guasch, 2016) en el arte reciente haya sido examinado en un importante corpus bibliográfico ${ }^{1}$, todavía escasean los e estudios específicos sobre prácticas colaborativas centradas en temas socioambientales. En concreto, estudiaremos el trabajo de artistas y colectivos como Carolina Caycedo, The Harrison Studio y Desert ArtLAB, actualmente activos en el continente americano. En este contexto geopolítico, caracterizado por formas históricas de colonialismo ambiental, económico y cultural, se han ido desarrollando en las últimas décadas, bajo la hegemonía politico-cultural de Estados Unidos, tipologiás de capitalismo cada vez más extremas. A partir de la desregularización de los años ochenta, se ha asistido, en efecto, a una constante erosión de los derechos, tanto sociales como ambientales, a la implementación de medidas económica neocoloniales y, en los últimos años, a la afirmación de gobiernos populistas de matriz ultraconservadora que han adoptado políticas reaccionarias en el campo de la protección de la naturaleza y de los derechos humanos e indígenas. En este panorama, las luchas y los movimientos contraculturales vinculados a la ecología, al feminismo y a la defensa de las culturas nativas de las Américas, junto a las expresiones culturales y artísticas que de ellos han brotado, se configuran como formas de resistencia frente a un modelo neoliberal cada vez más agresivo que se va consolidando a escala global.

\section{METODOLOGÍA}

Junto a la revisión bibliográfica de aportaciones teóricas de distintos ámbitos del conocimiento (teoría del arte, filosofía, ciencias naturales, etc.), el presente estudio se nutre de los resultados parciales de una estancia de investigación llevada a cabo en el Center for Creative Ecologies de la University of California Santa Cruz (EEUU). Para el análisis de las obras de arte seleccionadas se han utilizado métodos de investigación cualitativos típicos de las ciencias sociales, como la observación participante, entrevistas semiestructuradas con artistas, comisarios y otros colaboradores involucrados en los proyectos. El objetivo principal era indagar la intencionalidad de los creadores, su proceso creativo y las diferentes estrategias colaborativas empleadas para abordar y visibilizar las multiples dimensiones de las problemáticas socioambientales tratadas. Asimismo, nos parecía esencial adoptar un enfoque experiencial en la investigación. En efecto, como destaca el historiador del arte Grant Kester, las prácticas colaborativas exigen una ampliación de las herramientas analíticas de la crítica de arte debido a su carácter eminentemente procesual y colectivo. Según Kester,

\footnotetext{
${ }^{1}$ Vease al respecto To Life! Eco Art in Pursuit of a Sustainable Planet (Weintraub, 2012), Arte y Ecología (Raquejo \& Parreño, 2015) o Decolonizing Nature: Contemporary Art and the Politics of Ecology (Demos, 2016).
} 
para comprender estas formas de arte es necesario habitar el lugar donde se desarrollan "durante un período prolongado de tiempo, prestando especial atención a las condiciones discursivas, físicas y sociales del espacio, y a los ritmos temporales de los procesos que allí se desenvuelven" ${ }^{2}$ (Kester, 2013, p. 2). Siendo conscientes de la dificultad de llevar a cabo este tipo de estudio en todos los proyectos seleccionados, aplicamos esta metodología en el análisis de la serie de obras titulada Future Gardens de Helen y Newton Harrison durante nuestra estancia en Santa Cruz. Para las obras de los otros artistas citados, integramos las entrevistas con el estudio de la documentación foto y videográfica de los proyectos.

\section{DESARROLLO}

Si bien en sus vertientes más convencionales se encuentre inscrito de pleno en el sistema neoliberal, el arte contemporáneo todavía contiene espacios de oposición al paradigma cultural dominante no completamente colonizados por la lógica mercantilista. En efecto, las practicas creativas que se analizan es este estudio articulan posiciones críticas hacia el modelo capitalista generando una reflexión sobre sus impactos ambientales, éticos y sociales. Para valorar el potencial crítico de dichas prácticas, resultan particularmente significativas las aportaciones de Jacques Rancière sobre relación entre arte y política. En palabras del filósofo francés, "una intervención artística puede ser política modificando lo visible, las formas de percibirlo y expresarlo, de apreciarlo como tolerable o intolerable" (Carnevale, Kelsey, \& Rancière, 2007, p. 259). De este modo, el arte según Rancière puede transformer la "distribución y redistribución de los espacios y los tiempos, de los lugares y las identidades, de la palabra y el ruido, de lo visible y lo invisible" que conforman lo que el autor llama el reparto de lo sensible (Rancière, 2003, p. 16), un conjunto de convenciones mediante las cuales el sistema dominante modula las condiciones de visibilidad e inteligibilidad de todo hecho social. A partir de esta noción el filósofo distingue dos tipos de relaciones entre el arte y la sociedad que define repectivamente como régimen representativo y régimen estético. Los límites de esta investigación no nos permiten ahondar en los detalles de esta clasificación por lo que nos centraremos en el denominado régimen estético, donde el papel del arte es el de proporcionar espacios de disenso modificando "las coordenadas de lo sensible" (Carnevale et al., 2007, p. 259) y cuestionando las jerarquías normativas. Uno de los objetivos del presente estudio es analizar de qué manera las formas de arte colaborativo vinculado a la ecología contribuyen a repensar las condiciones de visibilidad de temáticas, cosmovisiones y sujetos normalmente situados al margen de los discursos hegemónicos. Asimismo, haciendo referencia a las recientes aportaciones del historiador del arte T. J. Demos, pretendemos mostrar como las diferentes prácticas artísticas estudiadas pueden "politizar la relación del arte con la ecología" (Demos, 2016, p. 8) y descolonizar las metodologías y los lenguajes artísticos para abordar la complejidad de la relación cultura-naturaleza desde una postura crítica hacia el antropocentrismo occidental.

Particularmente sugerente en este sentido es la poética de la artista colombiana Carolina Caycedo, cuyo trabajo reciente se centra en las dinámicas extractivistas que sustentan el modelo neoliberal analizando sus consecuencias sobre la salud de los ecosistemas naturales y de las comunidades que los habitan. El proyecto titulado Be dammed ${ }^{3}$ (2013-2018) se compone de una constellación de obras que pretenden generar una reflexión compartida sobre la construcción de macropresas para la generación de energía hidroeléctrica en el continente americano. Muchas de estas obras son el resultado de la colaboración de la artista con organizaciones indígenas activas en regiones de Colombia afectadas por estos proyectos de explotación y commodificación de los ecosistemas fluviales ${ }^{4}$. Empleando diferentes lenguajes artísticos como la instalación, el dibujo, el videoarte, la performance, etc., la artista visibiliza las alteraciones en la geografía, en el microclima y en los equilibrios ecológicos y sociales de los lugares interesados por la creación de estas grandes infraestructuras. Expone asimismo las contradicciones entre la retórica tecno-entusiasta que describe estas obras como un símbolo de progreso y desarrollo sostenible y la realidad de sus impactos nivel paisajístico y social. Al conectar las resistencias territoriales con reivindicaciones de justicia climática, social, cultural y de genero, Caycedo enfatiza no solo las implicaciones geopolíticas y económicas vinculadas a la creación de estas presas sino también las consecuencias más sutiles -y no por ello menos importantes- de estos procesos exctractivistas como la pérdida de habitats de elevada diversidad biológica, el desplazamiento forzoso de comunidades, la destrucción de lazos afectivos, de modos de vida tradicionales y de gran parte de la riqueza cultural ligada a la convivencia milenaria de las poblaciones locales con los ríos.

El carácter performativo del trabajo de Caycedo hace que el cuerpo humano se configure como herramienta de lucha ante estas politicas destructivas y al mismo tiempo como repositorio de conocimientos y espacio privilegiado para la percepción y comprensión del mundo que nos rodea. Por lo tanto, incluso cuando adquieren una dimension objetual, las obras de la creadora colombiana remiten al movimiento, a la activación de diálogos y gestos fuera de la lógica utilitarista. Por ejemplo, Serpent Book (2017), una publicación que ilustra los resultados de 5 años de investigación colaborativa sobre el tema de las represas y otras infraestructuras fluviales, es a la vez el registro de los procesos creativos llevados a cabo por la artista y un recurso poético para iniciar debates,

\footnotetext{
${ }^{2}$ Todas las traducciones, a menos que se indique lo contrario, son de la autora.

${ }^{3}$ El título es un juego de palabras entre los verbos ingleses to damn (maldecir) y to dam (represar).

${ }^{4}$ La gran mayoría de las presas en construcción en America Latina son controladas por empresas transnacionales que obtienen beneficios económicos a partir de un recurso común como es el agua. Una evaluación de los impactos socioambientales de las grandes infraestructuras hidroeléctricas puede encontrarse en "Sustainable hydropower in the 21st century" (Moran, Lopez, Moore, Müller, \& Hyndman, 2018).
} 
inspirar coreografías y acciones en entornos expositivos convencionales o en contextos cotidianos de lucha y socialización (Fig. 1 y 2). Esta dimensión corporeizada de la práctica aspira, en palabras de la misma creadora, a:

“...generar una empatía incorporada, una sensación física de cercanía que permita crear un vínculo emocional entre el público y algunos lugares y comunidades geograficamente y culturalmente distantes. Además, las obras representan una invitación a dejar de percibir el territorio como un paisaje separado de nosotros para aproximarnos a un cambio de paradigma, a una transición epistemológica que nos permita comprender que, como sostienen la mayoría de las cosmovisiones indígenas, el ser humano necesita de una multitud de otros seres -vegetales, animales, minerales, fenómenos atmosféricos, etc.- para existir. Y que la incapacidad de percibir y cultivar las conexiones materiales, políticas, espirituales y afectivas con nuestro entorno natural y social nos ha llevado a la situación de colapso climático que vivimos". 5
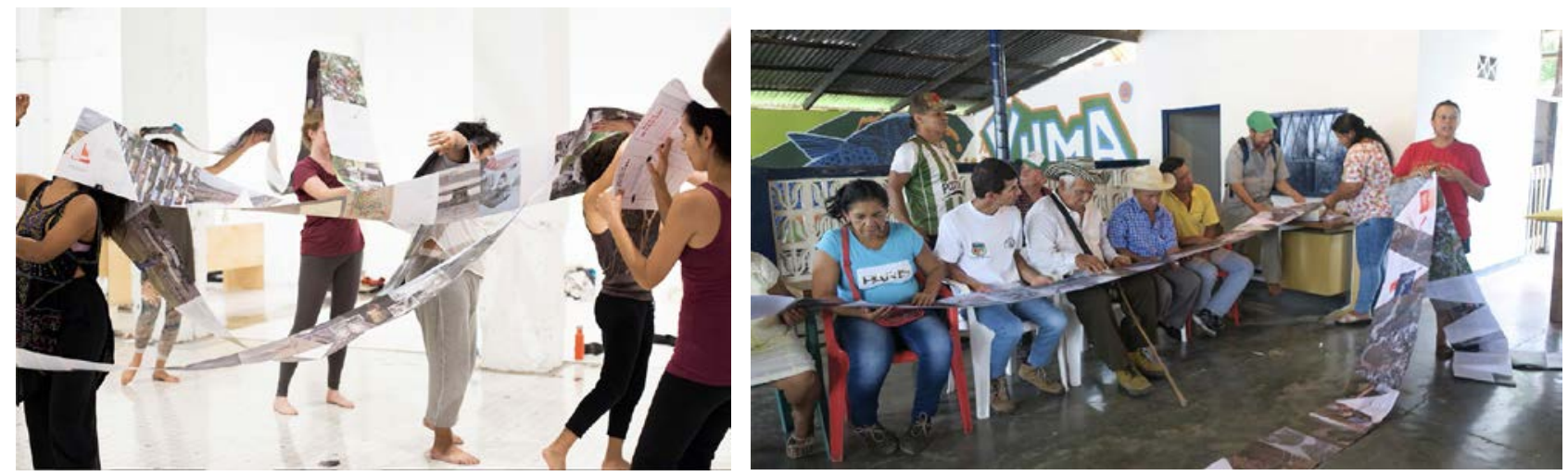

Figuras 1 y 2. Carolina Caycedo (2017). Serpent book. Libro de artista. 22 × 31 × 3.5 cm. 2017. Cortesía de la artista.

La investigación artística de Carolina Caycedo desvela las diferentes criticidades de una gestión del territorio basada en criterios extractivistas. Frente a la objetualización del mundo natural típica de la racionalidad capitalista, la artista intenta revitalizar los lazos de solidaridad entre cuerpos de agua, árboles, piedras, animales, seres humanos y comunidades, considerándolos como entidades con agencia e identidad propias. Asimismo, Caycedo contribuye a desarticular la jerarquía de los saberes, revalorizando, sin caer en la idealización, el papel de las sabidurías indígenas y de la creatividad artística en la búsqueda de posibles soluciones a los conflictos socioambientales.

Un marcado interés en la inclusion de otras epistemologías en los discursos artísticos vinculados a la ecología puede observarse también en el trabajo del colectivo interdisciplinar Desert ArtLAB, formado por la comisaria y educadora April Bojorquez y el artista y educador Matthew Garcia. La pareja, de ascendencia chicana y Rarámuri, deconstruye la visión del desierto como espacio inerte y hostil a la vida rescatando los conocimientos tradicionales de las culturas nativas del sur de Estados Unidos que prosperaron durante miles de años en ecosistemas áridos y semiáridos. Frente a las previsiones de desertificación de vastas áreas del continente americano y del mundo en general a causa de las alteraciones del clima, los artistas presentan el desierto como un lugar rico de recursos y de oportunidades de aprendizaje para la construcción de sociedades más ecológicas. Uno de sus proyectos más ambiciosos se titula Pueblo Field Site (de 2016 hasta la actualidad) y consiste en la transformación una parcela abandonada en el desierto de Colorado (EE $\mathrm{UU}$ ) en un paisaje comestible de plantas autóctonas. El proceso de recuperación de este terreno se ha llevado a cabo en colaboración con un grupo de estudiantes y otros miembros de la comunidad local. Tras despejar la zona de todos los residuos, se han plantado en el terreno varias especies vegetales autóctonas, sobre todo variedades de cactuses capaces de soportar las condiciones climáticas extremas del lugar. La presencia de estas plantas ha permitido restaurar en pocos años la ecología del suelo, favoreciendo el enraizamiento de otras especies vegetales y animales. Al respecto, los artistas afirman a que la motivación de la obra era:

"reconstruir un ecosistema indígena, recurriendo a conocimientos tradicionales presentes en nuestras familias y en las comunidades nativas de Arizona y Colorado, con el objetivo de descolonizar el concepto de naturaleza típico del imaginario norteamericano -basado en la presencia ambientes frondosos y exhuberantes- para visibilizar la estética de un paisaje vinculado a una noción de límite” ${ }^{6}$.

Además, Pueblo Field Site constituye de campo de experimentación para el cultivo de alimentos del desierto y la revitalización de práticas indígenas ligadas a la comida. En efecto, el libro Desertification Cookbook (2016) editado por Desert ArtLAB ilustra la gran variedad de flora y fauna que se se encuentra en regiones desérticas y que puede utilizarse para la alimentación humana. Las características botánicas, medicinales y ceremoniales de especies como el amaranto, algunas variedades de maíz, plantas crasas y

\footnotetext{
${ }^{5}$ Fragmento de la entrevista a Carolina Caycedo realizada por la autora el 13 de noviembre de 2018 en Santa Cruz (California, Estados Unidos).

${ }^{6}$ Entrevista a April Bojorquez y Matthew Garcia (Desert ArtLAB) realizada por la autora el 27 de enero de 2018 en San Francisco (California, Estados Unidos).
} 
muchas otras son descritas en esta publicación junto a sus vínculos con las mitologías y las creencias nativas. La regeneración de este patrimonio de saberes conecta la cuestión de la ecología con la justicia social, planteando una estrategia de autodeterminación y reapropiación cultural (Hanley, 2018) para unas poblaciones que han sido sometidas e invisibilizadas por la colonización europea. Estas y otras obras del colectivo han sido expuestas en centros culturales, museos, galerías y en espacios públicos. Casi siempre son acompañadas por actividades educativas intergeneracionales, performances, talleres y clases de cocina que pretenden crear momentos de aprendizaje comunitario que movilicen la memoria de las culturas indígenas en conexión con los desafíos del presente para explorar otras sensibilidades y otros modos de estar en el mundo.

La formulación de respuestas pragmáticas y poéticas frente a los problemas ecológicos es, junto al diálogo transdisciplinar, uno de los aspectos fundamentales de la producción artística de Helen Mayer y Newton Harrison. Pioneros en la experimentación con procesos colaborativos de restauración de ecosistemas y en la ideación de modelos ecocompatibles de gestión de los recursos naturales, los Harrison han contribuido desde finales de los años sesenta a expandir las fronteras del arte inspirado en principios ecológicos. Entre sus proyectos más recientes destaca la serie Future Gardens (de 2011 hasta la actualidad), compuesta por tres jardines en los que se cultivan plantas que podrían conformar los ecosistemas del mañana. El primero se creó en la localidad de Sagehem (California), otro se implantó en Tibet y el más reciente se encuentra en el jardín botánico de la University of California Santa Cruz. La visita a este último y la realización de una entrevista con el artista Newton Harrison han permitido indagar las distintas fases del proceso de creación del proyecto, sus principales objetivos y características estéticas. La obra, titulada Future Garden for the Central Coast of California (2018), consta de una instalación site-specific compuesta por tres cúpulas geodésicas convertidas en invernaderos y constituye un experimento artístico y científico a la vez en respuesta a la crisis del cambio climático. Para su realización, Newton Harrison ${ }^{7}$ ha trabajado con botánicos y climatólogos de la universidad de Santa Cruz para seleccionar dieciséis especies vegetales autóctonas de máxima resiliencia con el fin de estudiar su capacidad de adaptación. Además, junto a otros artistas y estudiantes, ha diseñado el jardín y acondicionado las cúpulas para reproducir las condiciones climáticas de humedad, precipitaciones y temperatura que, según las previsiones, caracterizarán la costa central de California en los próximos años. El objetivo de la investigación es determinar qué plantas son más capaces de prosperar a medida que la región se calienta y, en una segunda fase, propagar dichas especies para favorecer una regeneración más rápida del ecosistema local en respuesta a las nuevas y más extremas condiciones del clima.

Sin embargo, la instalación no representa solo un dispositivo útil para recopilar datos, acumular conocimientos botánicos y hacer visible los efectos de las alteraciones climáticas en el reino vegetal. En efecto, la obra conforma un recorrido contemplativo, un espacio de proximidad y reflexión sobre la relación de interdependencia que vincula seres humanos y plantas. Un lugar de observación, escucha y cuidado donde "prestar atención" (De Certeau, 1985, p. 17) a los procesos naturales es un acto transformador. Entre los elementos innovadores de este proyecto es de reseñar la transición de un enfoque basado en estrategias de reparación de entornos antropizados, típica de los trabajos iniciales de los Harrison, hacia la experimentación con una ecología que podríamos definir como preventiva, cuya finalidad es anticipar y facilitar la coevolución de distintas especies para construir ecosistemas resilientes para el futuro. Particularmente interesante es también el carácter replicable de este proyecto: en efecto, esta metodología es susceptible de ser repetida y aplicada libremente en otros lugares para contribuir a la búsqueda de especies resistentes al cambio climático en distintos contextos geoclimáticos y socioculturales. Lejos de apostar por soluciones tecnológicas y de geoingeniería ${ }^{8}$ a menudo vinculadas al capitalismo verde, el proyecto Future Gardens propone una estrategia accessible, relativamente barata, basada en la naturaleza y en la composición de saberes diversos. La elección de ensamblar distintas especialidades y perspectivas surge, según Newton Harrison, de la necesidad de "construir un conocimiento generalista y elaborar soluciones sistémicas" 9 para encarar fenómenos globales e interconectados como el calentamiento del planeta o la sexta extinción masiva, frente a los que la hiperespecialización de la ciencia moderna se ha mostrado insuficiente.

\footnotetext{
${ }^{7}$ Tras casi 50 años de colaboración, esta obra es la primera en la que el artista trabaja sin su esposa Helen Mayer, fallecida en agosto de 2018.

${ }^{8}$ Un análisis de la relación entre tecno-utopías, geoingeniería y capitalismo en la época del llamado Antropoceno puede encontrarse en el ensayo "To Save a World: Geoengineering, Conflictual Futurisms, and the Unthinkable" (Demos, 2018).

${ }_{9}^{9}$ Fragmentos de la entrevista a Newton Harrison realizada por la autora el 6 de diciembre de 2018 en Santa Cruz (California, Estados Unidos).
} 

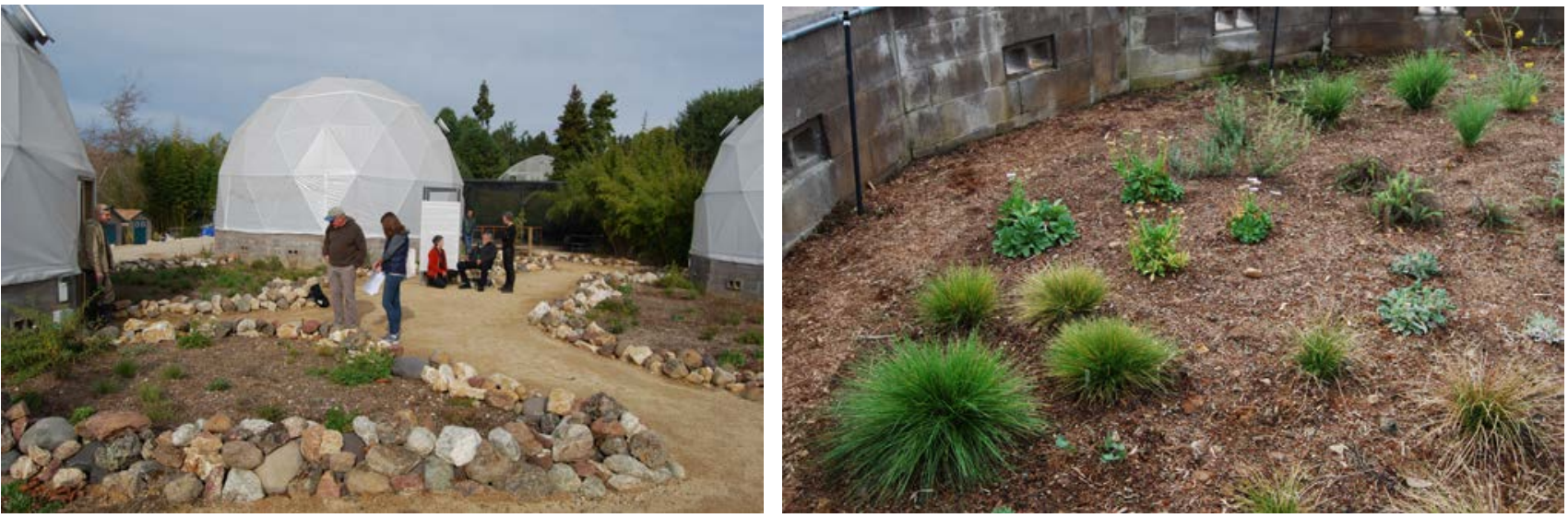

Figuras 3 y 4. Harrison Studio (2018). Future Garden for the Central Coast of California. Visita guiada con el artista Newton Harrison y detalle de las plantas que componen la instalación. Fotografía: Chiara Sgaramella.

\section{CONCLUSIONES}

Aunque diversas en contenidos y resoluciones formales, las prácticas artísticas analizadas hacen perceptibles mediante la experiencia estética procesos ecosociales complejos, a menudo dilatados en el tiempo y normalmente excluídos de los discursos culturales dominantes. Su relevancia política no reside unicamente en las dimensiónes discursivas de las obras sino también en los métodos empleados para su creación. En efecto, estos proyectos articulan formas de disenso "reconstruyendo la relación entre lugares e identidades, [...] proximidades y distancias" (Carnevale et al., 2007, p. 261) y al mismo tiempo adoptan fórmulas cooperativas y horizontales de creación, tejiendo alianzas transdiciplinares, interespecie e intergeneracionales. Esta dimensión colaborativa permite incorporar una pluralidad de miradas en el abordaje de asuntos complejos como los que plantea la crisis climática contemporánea, incluyendo perspectivas éticas y epistemológicas alejadas del paradigma antropocéntrico occidental. Además, las manifestaciones artísticas estudiadas activan nuevas formas de compromiso social y ambiental fortaleciendo los vínculos de resiliencia a nivel social y ecosistémico. Al mismo tiempo pueden interpretarse como ejercicios de imaginación crítica que no solo contribuyen a reconfigurar el reparto de lo sensible sino que reinventan lo que Rancière llama "el paisaje de lo" (Carnevale et al., 2007, p. 264) ofreciendo la posibilidad de "vislumbrar la belleza de vivir de otra manera" (Demos, 2016, p. 272).

\section{FUENTES REFERENCIALES}

Carnevale, F., Kelsey, J., \& Rancière, J. (2007). Art of the Possible: Fulvia Carnevale and John Kelsey in conversation with Jacques Rancière. Artforum, (March), 256-269.

De Certeau, M. (1985). Pay Attention: To Make Art. In H. Mayer \& N. Harrison (Eds.), The Lagoon Cycle (pp. 17-23). Ithaca: Herbert F. Johnson Museum of Art, Cornell University.

Demos, T. J. (2016). Decolonizing nature: contemporary art and the politics of ecology. Berlin: Sternberg Press.

Demos, T. J. (2018). To Save a World: Geoengineering, Conflictual Futurisms, and the Unthinkable. E-Flux, (94). Disponible en: https://www.e-flux.com/journal/94/221148/to-save-a-world-geoengineering-conflictual-futurisms-and-the-unthinkable/

Guasch, A. M. (2016). El arte en la era de lo global 1989-2015. Madrid: Alianza Editorial.

Hanley, A. R. (2018). Lands between Us Lands Apart. In Trees Also Speak. Old Westbury: Amelie A. Wallace Gallery, SUNY College.

Herrero, Y. (2013). Miradas ecofeministas para transitar a un mundo justo y sostenible. Revista de Economía Crítica, 16, $278-307$. Disponible en: http://www.revistaeconomiacritica.org/sites/default/files/revistas/n16/09 YayoHerrero.pdf

Kester, G. (2013). Collaborative Arts and the Limits of Criticism. Create, (14). Disponible en: http://www.createireland.ie/images/pdfs/create-news/Grant-Kester-Collaborative-Art-and-the-Limits-of-Criticism-CREATE-NEWS-14-May-2013.pdf 
Masson-Delmotte, V., Zhai, P., Pörtner, H.-O., Roberts, D., Skea, J., Shukla, P. R., ... Waterfield, T. (2018). Global warming of $1.5^{\circ} \mathrm{C}$. An IPCC Special Report on the impacts of global warming of $1.5^{\circ} \mathrm{C}$ above pre-industrial levels and related global greenhouse gas emission pathways, in the context of strengthening the global response to the threat of climate change. Disponible en: https://www.ipcc.ch/site/assets/uploads/sites/2/2018/07/SR15 SPM version stand alone LR.pdf

Moran, E. F., Lopez, M. C., Moore, N., Müller, N., \& Hyndman, D. W. (2018). Sustainable hydropower in the 21st century. Proceedings of the National Academy of Sciences of the United States of America, 115(47), 11891-11898. https://doi.org/10.1073/pnas.1809426115

Rancière, J. (2003). Política de la literatura. Buenos Aires: El Zorzal.

Raquejo, T., \& Parreño, J. M. (Eds.). (2015). Arte y ecología. Madrid: Editorial UNED.

Riechmann, J. (2011). Vivamos bien. En ¿Cómo vivir? Acerca de la vida buena (pp. 7-66). Madrid: Los Libros de la Catarata.

Weintraub, L. (n.d.). To life! Eco art in pursuit of a sustainable planet (2012th ed.). Oakland: University of California Press. 